\title{
Study on Mechanical Property of Corroded Pipeline
}

\author{
FAN YINGFNAG HU ZHIQIANG ZHOU JING \\ State Key Laboratory of Coastal and Offshore Engineering, \\ Dalian University of Technology \\ Dalian, Liaoning \\ P.R. China
}

\begin{abstract}
Stochastic finite element method (FEM) was applied to analyze the mechanical property of the corroded pipeline. Firstly, mechanical properties of the corroded steel were studied. Then, dynamic properties of the corroded pipeline were investigated; sensitivity analyses were performed on the effective factors respectively. Considering the distribution characters of the defects on the pipe surface as stochastic variables, a series of numerical investigations were executed on the corroded pipeline. Relation between the dynamic property of the submarine pipeline and corrosion state of the material is achieved.
\end{abstract}

Key-Words: - Corroded pipeline; mechanical property; statistical distribution function; stochastic FEM; factor analysis

\section{Introduction}

The construction of submarine pipelines for delivering gas and oil is increasing with the exploration and utilization on the offshore oil/gas resource in China. However, since salt content of seawater is about 3 percent, and the seawater is a kind of strong electrolyte, pipelines servicing in the marine environment will be corroded by the seawater and the marine atmosphere. Furthermore, Sulfur dioxide and carbon dioxide will take corrosive effects on the structures as well. Meanwhile, many environmental loads acting on the pipelines, such as wave, flowing of the fluid etc., will accelerate the corrosion effects on the deterioration of the structure. Although some methods (such as anticorrosion coating below the concrete coating and catholic protection using sacrificial anodes) have been accepted to protect the modern pipeline from corrosion, submarine pipelines in service always showed early deterioration. By now, the failure of the submarine pipeline has led to the broken-down of the oilfield production, and has caused the pollution of the surrounding sea area. Therefore, as a large submarine transport network, safety of the pipeline has become to be a critical issue and attracted more and more attention by scientists and engineers worldwide, and the evaluation of the integrity of the corroded pipeline has long been and continuous to be a concern.

Since 1970s, improved condition assessment methods for the pipeline systems have been continuously seek for. The original work was sponsored by AGA-NG18 in early 1970, and a semi-empirical equation for remaining strength of corroded pipelines was developed by Battelle (Bjornoy and Marley,2001). Modifying the formula proposed by Battelle, some other methods such as Shell criterion, RSTRENG, etc have been suggested. However, both Battlle's formula and the various modified methods are just taking the single corrosion metal mass loss defects in pipelines exposed to internal pressure into account, regardless of the interaction of defects and additional loads, etc. Based on the risk and reliability principles, Nes(2001) established a methodology for pipeline condition assessment.

To develop a rational evaluation of the present state of the submarine pipeline, it is essential to make a reliable study on the mechanical property of the deteriorating structures. The mechanical property of the corroded submarine pipelines has been investigated ( $\mathrm{Fu}$ 1995,Batte 1997, Moke 1991,Zhu 2003). In a summary, two aspects of deficiencies exist in their studies: 1) Since the corrosion is a stochastic process, both the mechanical property of the material and the structure were always affected by many stochastic factors, such as, the corrosive medium around the pipeline, corrosion defects on the metal (such as depth, number and the distribution law of the defects, et al), and the additional loads, etc. However, the stochastic character has not been taken into account in the present studies. 2) For the corroded in-service submarine pipeline, elastic stage will be shortened and the structure will enter into plastic stage earlier during the loading process, which will result in the early occurrence of surface cracking, and the cracking of the concrete will accelerate the corrosion of the metal. Since the effective factors are 
complex, and coupling effect always exists among the factors, it is difficult to take all the factors into account simultaneously. The present studies are limited to single metal loss defect, and excludes interaction of the defects and other loads.

In this paper, stochastic finite element method (SFEM) will be applied to analyze the mechanical property of the corroded pipeline. Firstly, the effects of the defects on the steel were studied. Taking characters of the defects on the metal as stochastic variables, a series of numerical investigations of corroded submarine pipelines were performed. The changing tendency of the dynamic property of the submarine pipeline with the corrosion state of the material is achieved.

\section{Mechanical Property of the rusty steel}

\subsection{Steel corrosion character in concrete}

Corrosion mechanism of the steel in concrete is an electrochemistry process, which had been studied widely. To describe the corrosion state of the steel in concrete, a kind of NDT instrument (GXY-1A) was applied for the corroded reinforced concrete beams (depth of the concrete cover is $20 \mathrm{~mm}$ ), which had been corroded severely by Chloride for more than 10 years. A potential map of RC beam is shown in Fig.1, in which the figures indicate the potential value of the measuring point. If the potential value bellows -400 , it is indicated that the reinforcement in the area have been severely corroded.

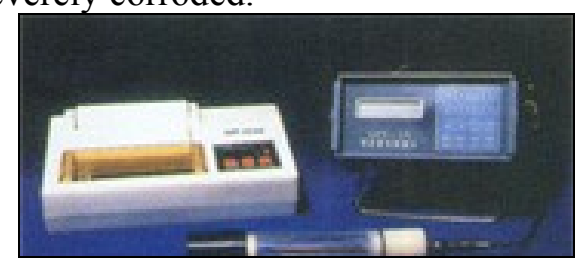

Fig.1 NDT instrument(GXY-1A )

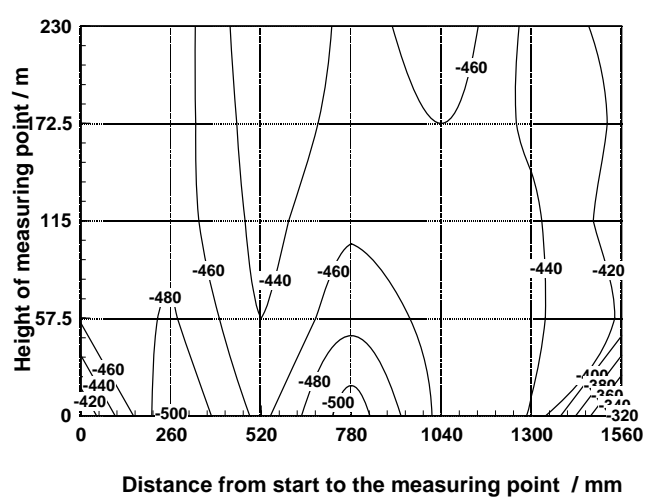

Fig.2 Potential maps of RC beam corroded by Chloride
From Fig.2, it is shown that chloride has a strong corrosive effect on the RC structure, and lead to occurrence of the corrosion pit on the steel surface.

\subsection{Mechanical property of the defected steel} The commercial finite element software, ANSYS, was applied to study the mechanical property of the steel. 2D finite element model of the steel with a diameter of $18 \mathrm{~mm}$ is shown in Fig.3. Values of $340 \mathrm{MPa}, 520 \mathrm{MPa}, 200 \mathrm{Gpa}$ and 0.3 were used for the yield strength, the ultimate strength, Modulus of Elasticity and Poisson's ratio respectively in the analyses. Idealistic elastic-plastic constitutive model of the steel is applied in the analysis, which is shown in Fig.4.

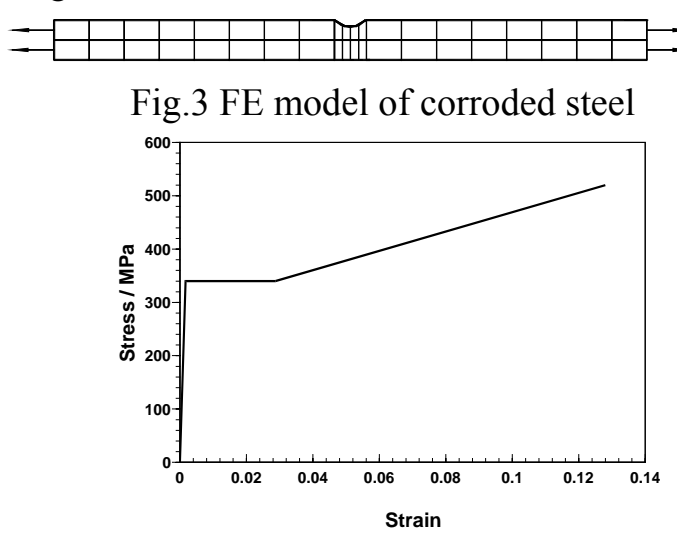

Fig.4 Constitutive model of the steel

Assuming that there exists a corrosion pit with the depth of $1 \mathrm{~mm}$ on the steel surface, the stress distribution along the longitudinal direction under the yield load of the intact steel can be obtained in Fig.5.

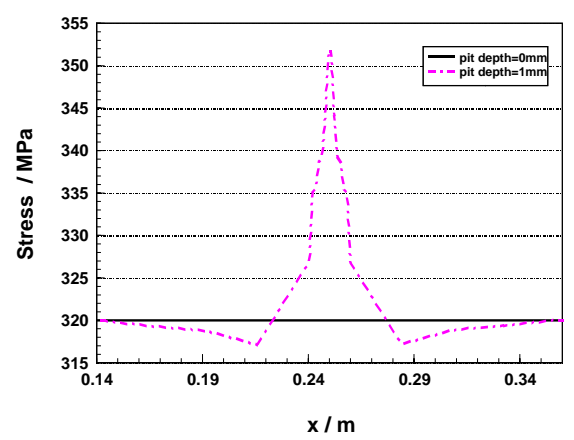

Note: $\mathrm{x}$ is the distance from the corrosion pit to the end of steel Fig.5 Stress distribution along the tensile direction

\subsection{Sensitivity analysis of the effective factors}

According to the corrosion mechanism of the steel in concrete, the corrosion pit always takes on 3 types of shape: shallow, intermediate and deep corrosion defects. The mechanical property of the damaged steel is always affected by many factors, such as the 
shape, location and maximum depth of the corrosion pit, distribution and the number of the maximum corrosion pit, the steel diameter, etc. Sensitivities of these parameters on the mechanical property of the damaged steel are investigated respectively as follows.

\subsubsection{Effect of the pit shape on the mechanical property of the defected steel}

Considering the corrosion pit on the steel surface with the width change from 4 to $50 \mathrm{~mm}$ and the depth change from 1 to $7 \mathrm{~mm}$, the yield load of the defected steel can be numerated respectively. Therefore, the relationship between the pit shape (width and depth) and the normalized ultimate load $\mathrm{P} / \mathrm{P} 0$ ( $\mathrm{P}$ and $\mathrm{P} 0$ are the yield load of the defected and the intact steel respectively) can be achieved (Fig.6).

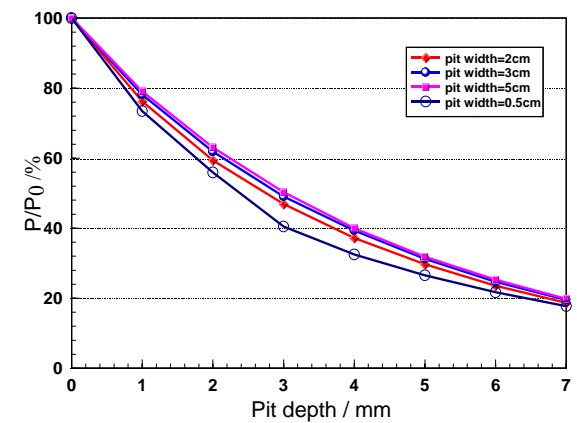

(a) Effect of the depth of corrosion pit on the yield load of rebar

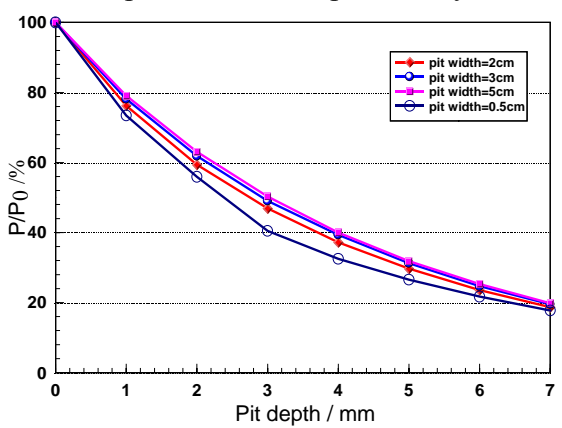

(b) Effect of the depth of corrosion pit on the yield load of rebar

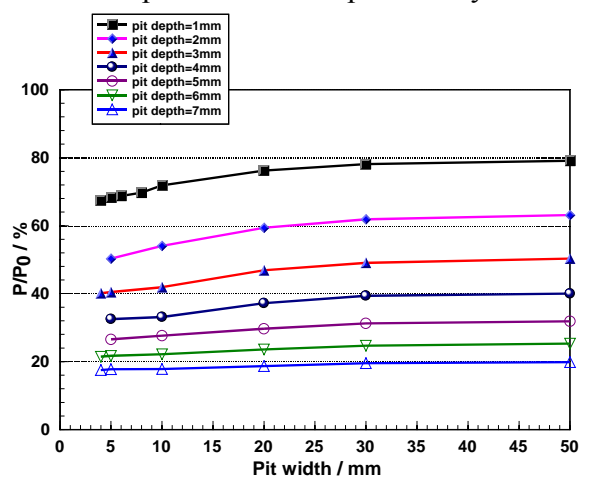

(c) Effect of the width of corrosion pit on the yield load of rebar Fig. 6 Effect of corrosion pit on the yield load of defected steel

From Fig.6, it can be concluded that the degradation of the defected steel is mainly determined by the depth of corrosion pit, while the width of the corrosion pit have a relative little effect on the mechanical property of the damaged steel.

\subsubsection{Effect of the maximum pit location on the mechanical property of the defected steel}

Since that the maximum pit depth is the key effective factor, effect of the location of the maximum pit on the defected steel is discussed herein. Considering the depth of the pit range from 0.5 to $7 \mathrm{~mm}$, the location of the pit move from the end of the steel to the middle of the steel, the yield load of the steel can be numerated respectively. The relationship between the pit locations versus the normalized ultimate load can be obtained.(Fig.7)

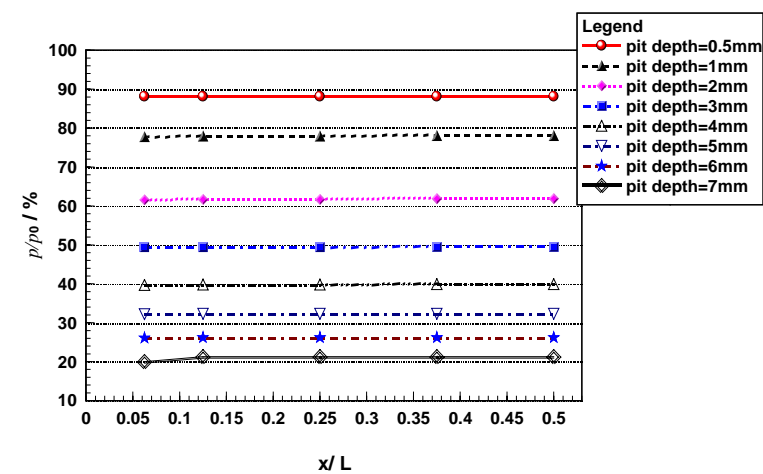

Note: $\mathrm{x}$ is the distance from the corrosion pit to the end of steel; $\mathrm{L}$ is the length of the steel

Fig.7 Effect of the pit location on the yield load of steel

The result shows that the pit location has a minor effect on the yield strength of the defected steel.

\subsubsection{Effect of the number of the pit on the mechanical property of the defected steel}

To study the effect of the number of corrosion pits on its mechanical property, the defected steel with 1 to 4 pits (the pit depth is $0.5,1,2,3,4,5,6$ and $7 \mathrm{~mm}$ ) were numerated respectively. Relationship between the pit number and the normalized ultimate load is plotted in Fig.8.

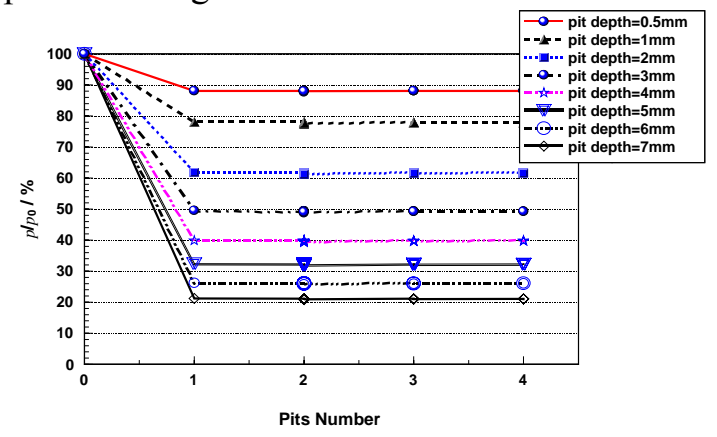

Fig. 8 Effect of the number of pit on the yield load of steel 
From the numerating results, it is evident that that the pit number have little effect on the load strength of the defected steel From the numerating results, it is evident that that the pit number have little effect on the load strength of the defected steel

\subsubsection{Effect of the steel diameter on the mechanical property of the defected steel}

Steel diameter is another effective factor for the mechanical property of the defected steel as well. Steels with 6 diameters $(14,16,18,20,22,25 \mathrm{~mm})$, and the pit depth is $1,2,3,4,5 \mathrm{~mm}$ for each kind of steel were analyzed respectively. Relationship between the steel diameter and the normalized ultimate load can be obtained under each pit depth (Fig.9 (a)). Furthermore, the relationship between the ratio of pit depth to diameter and the normalized ultimate load is given in Fig.9 (b).

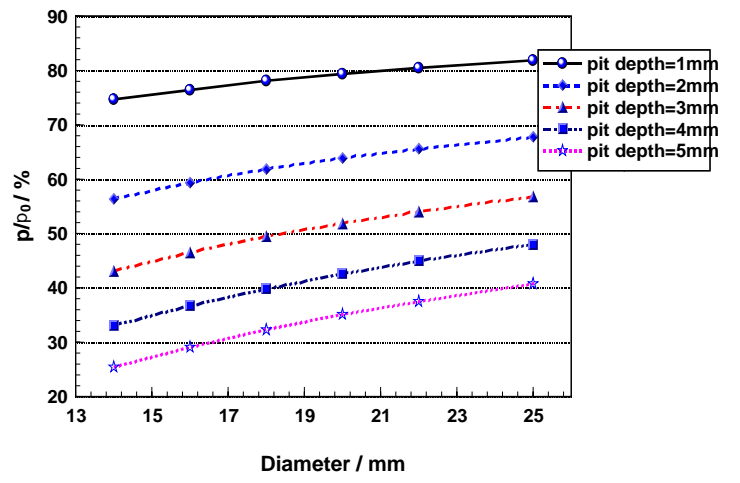

(a) relation between steel diameter and the $\mathrm{P} / \mathrm{P}_{0}$

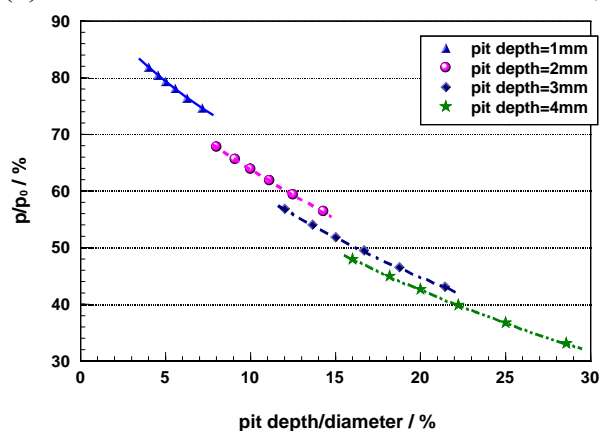

(b) relation between steel diameter and the $\mathrm{P} / \mathrm{P}_{0}$

Fig.9 Effect of the steel diameter on the yield load of steel

From the above, it can also be derived that with the deeper of the corrosion pit; relation between the yield load and the relative depth will be closer to be linear.

Therefore, relation between the yield load and the relative depth of the rusty rebar (pit depth/rebar diameter) can be derived,

$$
p=\left[a_{1}-a_{2} x+a_{3} x^{2}\right] \cdot p_{0}
$$

Where $\mathrm{p}$ is the yield load for the rusty rebar, $\mathrm{p}_{0}$ is the yield load for the sound rebar, $a_{1} 、 a_{2}$ and $a_{3}$ are the effective factors; which are relative to the maximum pit depth.

Table 1 Calculated value of the factors for different corrosion pit depths

\begin{tabular}{cccc}
\hline Pit depth $/ \mathrm{mm}$ & $a_{1}$ & $a_{2}$ & $a_{3}$ \\
\hline 1 & 94.0417 & 3.454 & 0.10368 \\
2 & 85.9302 & 2.514 & 0.03144 \\
3 & 81.212 & 2.357 & 0.02709 \\
4 & 75.25578 & 1.99703 & 0.01828 \\
\hline
\end{tabular}

\section{Stochastic analysis of the defected steel}

Using the experimental data of Aziz, which Al alloy corroded in the water for 1 week, 3 months, 1 year respectively, Finleg derived the probabilistic distribution rule of the maximum corrosion pit depth (Fig. 10(a)). From the corrosion experiment of alloy of Al-Mg in 3.5\% solution of $\mathrm{NaCl}$, Xiao (1994) applied statistical method analyze the distribution rule of the maximum pit depth on the alloy surface (Fig. 10(b)). It is discovered that the distribution of the maximum depths of pits accords with the Gumbel first approximating function. The extreme value law can be aptly used to evaluate pit corrosion problems. Probabilistic distribution function can be expressed as,

$$
F(x)=\exp \{-\exp [-(x-\mu) / \sigma]\}
$$

where $\mu$ is the location factor, $\sigma$ is the scalar factor, $x$ is the maximum pit depth.

However, lacking of experimental data led to the high difficulty to determine the location factor and scalar factor exactly by statistical analysis up to now. In this paper, the mean value $\mu$ and the standard deviation $\sigma$ are assumed to be $6.7 \mathrm{~mm}$ and $0.8 \mathrm{~mm}$ respectively. A sample set with a sample size of 750 was generated by Matlab, the probabilistic density distribution of the sample is shown in Fig.11 (a). Statistical distribution of the maximum stress along the steel can be achieved (Fig. 11 (c)).

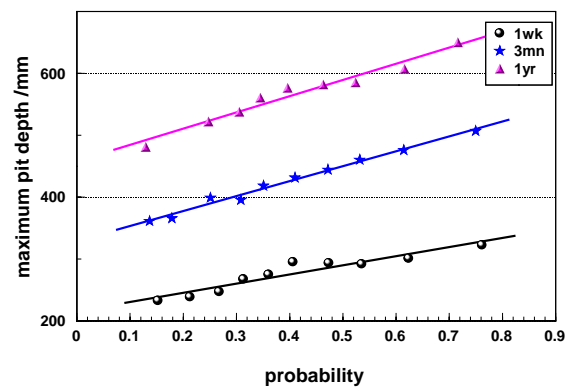

(a) corrosion of Al Alloy in water 


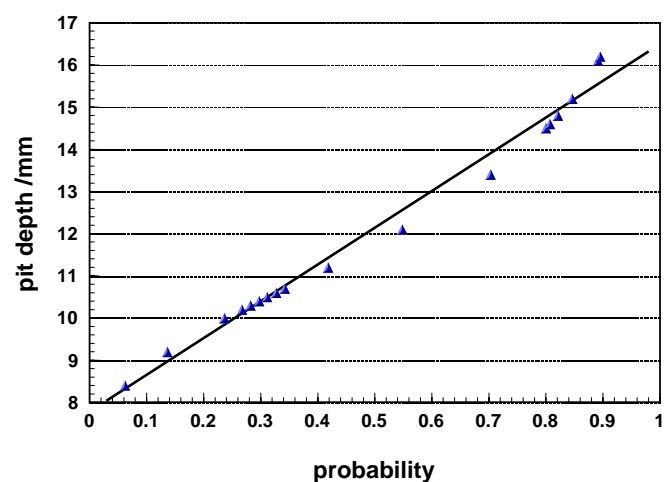

(b) corrosion of Al-Mg Alloy in $\mathrm{NaCl}$

Fig.10 Probabilistic distribution function of the maximum corrosion pit depth on metal surface

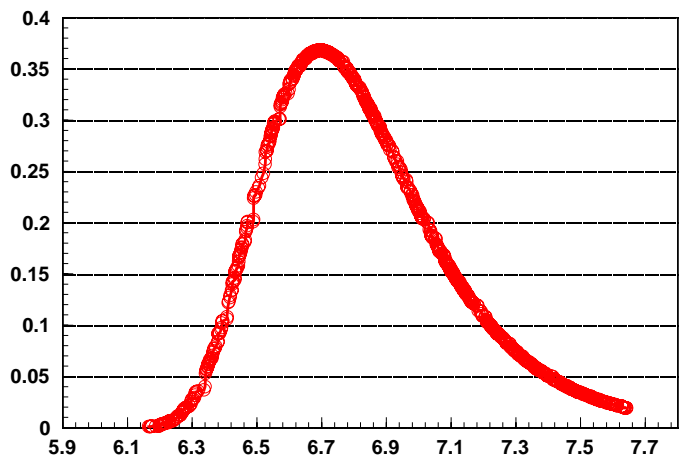

(a) probabilistic density distribution of the maximum pit depth

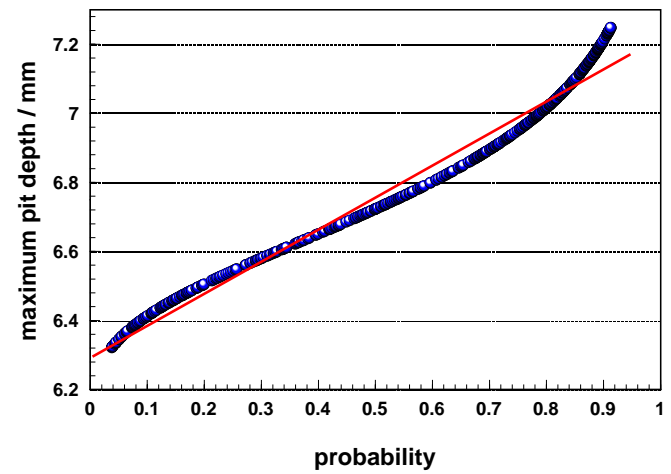

(b) probabilistic distribution of the maximum pit depth

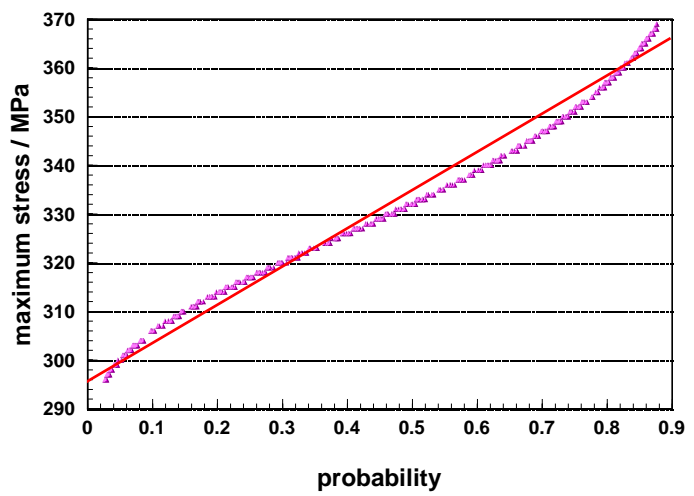

(c) probabilistic distribution of the maximum stress

Fig.11 Probabilistic distribution of the maximum corrosion pit depth and maximum stress
From the numerating results, it is indicated that if the maximum depth of pits obeys to extreme value law, the distribution of the maximum stress of the rebar will accord with the Gumbel first approximating function. The probabilistic distribution function $\mathrm{F}(\mathrm{x})$ can be written as,

$$
F(x)=\exp \{-\exp [-(x-324.13) / 21.93]\}
$$

Where, $F(x)$ is the probabilistic distribution function, and $\mathrm{x}$ is the maximum stress of the rebar.

\section{Mechanical Property of the corroded pipeline}

\subsection{Corrosion description of the submarine pipeline}

According to the design discipline of the submarine pipeline, the steel pipe is covered by concrete. The steel pipe is the main part for load bearing, while the concrete is the secondary part. Cross section of the pipeline is plotted in Fig.12. Interactive action of electrochemistry and the complex environmental loads are the mean causes for the corrosion of the pipelines.

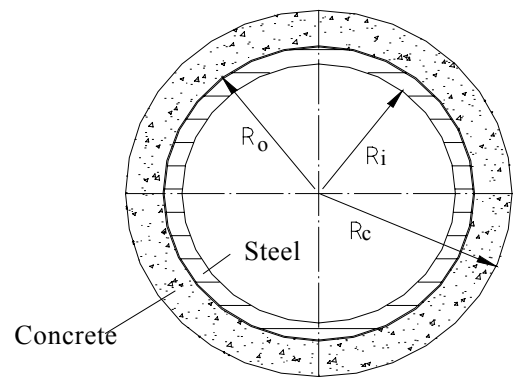

Fig. 12 Cross section of the pipeline

\subsection{Numerical model}

Considering that steel is the major part for the load bearing, and corrosion of the steel is the key effective factor for the mechanical property of the pipeline, the concrete is not taken into consideration herein. In this study, geometry of the pipeline is with an outside diameter of $508 \mathrm{~mm}(20 \mathrm{in}$.) and average wall thickness of $6.35 \mathrm{~mm}$ ( $0.25 \mathrm{in}$.). Ideal elastic-plastic constitutive model of the steel, which is shown in Fig.3, is applied in this analysis as well. The commercial finite element software, ANSYS, was applied to study the dynamic mechanical property of the pipeline. The 3D FE numerical model of the pipeline is shown in Fig.13.

According to the corrosion mechanism of the steel in concrete, the corrosion of the steel pipe is developed from a corrosion pit. Assuming that there exists a corrosion pit on the steel surface, the dynamic property of the pipeline will be studied. 
Different defect shape are considered, ratio of the defect depth to the pipe wall thickness varies from 0.1 to 0.5 , ratio of the defect width to the defect width varies from 2 to 10 .

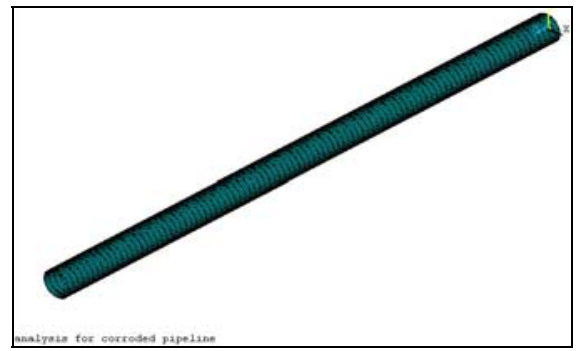

(a) FE model of the pipeline

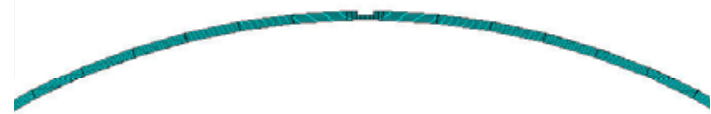

(b) defect on the surface of the steel

Fig.13 Numerical model of pipeline

\subsection{Sensitivity analysis of the effective factors}

\subsubsection{Effect of the pit shape on the load carrying capacity of the defected pipeline}

Considering the corrosion pit on the pipe surface with the depth change from $1 / 4$ to $1 / 2$ of the pipe thickness and the width change from 4 to $8 \mathrm{~mm}$, the yield load of the defected steel can be numerated respectively. Relationship between the pit shape (width and depth) and the normalized ultimate load $\mathrm{P} / \mathrm{P}_{0}\left(\mathrm{P}\right.$ and $\mathrm{P}_{0}$ are the yield load of the defected and the intact steel respectively) can be achieved. It can also be drawn that it can be drawn that the maximum pit depth is the key effective factor to the mechanical degradation of the damaged pipeline.

\subsubsection{Effect of the defect shape on the dynamic property of the defected pipeline}

Since dynamic loads (such as wave, fluid, earthquake, etc) are the major loads acted on the pipeline, the dynamic mechanical property of the pipeline is investigated in this paper. Based on the finite element model shown in Fig.13, taking the defect width and depth as stochastic variables, the dynamic responses for different free spanning length $(20 \mathrm{~m}, 60 \mathrm{~m}, 90 \mathrm{~m}$, $120 \mathrm{~m})$ in simply supported end condition are calculated. Assuming that the mean value $\mu$ and the standard deviation $\sigma$ of the pit depth are $0.02 \mathrm{~mm}$ and $2 \mathrm{~mm}$, while the values of $\mu$ for the pit width are $2 \sim 10$ times to the value of pit depth, a sample set with a sample size of 1000 was generated with direct Monte Carlo Simulation method. Probabilistic distribution of the corrosion pit depth is shown in Fig.14. Length of the defect along the longitudinal direction ranges from 5 time of the defect width to the length of the pipe. Modal analysis was performed respectively, and the frequencies of the structure can be extracted. Statistical distribution of the structural frequencies of the pipeline can be achieved.

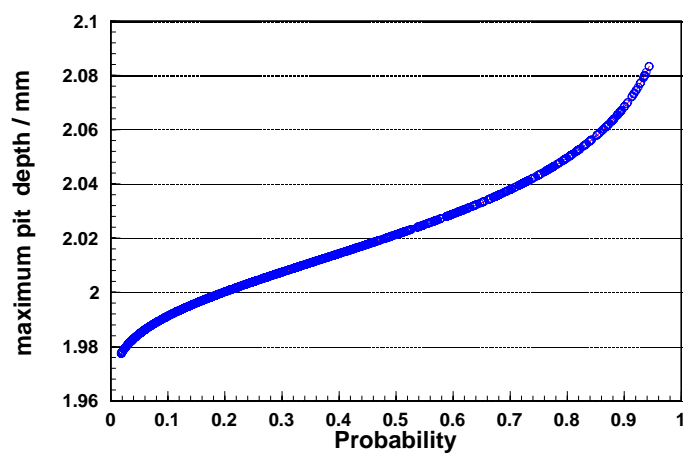

Fig.14 Probabilistic distribution of the corrosion pit depth

The results for each length of the defect show a similar trend, that is, both the single defect shape and the length of the pipe have minor effect on the natural frequency of the damaged structure. Even if the defect runs through the whole pipe, just a slight change occurs on the structural frequency, which is less than $0.5 \%$.

\subsubsection{Effect of the defect location on the dynamic property of the defected pipeline}

Considering the depth of the pit range from $1 / 10 \sim 1 / 2 t$, the location of the pit rotate from the top of the pipe to the bottom of the pipe, and the pit move from the end to the middle of the pipe. Modal analysis was executed on the defected model respectively, and the structural frequencies were achieved. All the results show the same tendency, that the frequency has little change with the different defect location.

From the above study, it can be drawn that neither the location nor the quantity of the damage has distinct effect on the structural frequency.

\section{Conclusion}

Taking a kind of corrosion measuring instrument (GXY-1A), corrosion state of the steel in concrete is depicted. Sensitivities of the factors (such as the width, the depth, the location, the number of the corrosion pit, etc) on the mechanical property of the damaged steel are analyzed respectively. Simulating the corrosion as a pit on the steel surface, numerical investigation is carried out on the corroded pipeline in this paper. From the results, the following conclusions were drawn: 
(1) The corrosion pit is the key factor that results in the degradation of the damaged steel;

(2) From the sensitivity analysis of the effective factors for the mechanical properties, it can be drawn that the maximum pit depth is the key effective factor to the mechanical degradation of the damaged steel, while the width, location, and number of the pit all have a minor effect on the mechanical property of the damaged steel;

(3) Taking the maximum depth of the corrosion pits as random input variable, and assuming that the distribution of the pit depth obeys to normal distribution, the maximum stress is numerated by SFEM. From the numerating results, it is indicated that the maximum stress will obey to normal distribution if the pit depth obeys to normal distribution;

(4) Taking both the maximum depth and the width of the corrosion pit as random input variable, it is concluded that the single defect shape has minor effects on the natural frequency of the damaged pipeline.

From the numerating results, it is discovered that the pipe length, the defect location and the defect number all take minor effects on the natural frequency of the damaged pipeline. As a result, it will be difficult to fulfill the health condition assessment of the in-service pipeline by the structural frequency obtained from the dynamic monitoring

\section{Acknowledgements}

This paper is partially funded by a National Funded project (Grant No.50378012), and a National Doctoral Funded project "Study on Failure Mechanism of Submarine Pipelines Under Corrosion and Loadings".

\section{References:}

[1] Hroar Nes and Lars Sortland, Risk-based condition assessment and inspection planning for submarine pipeline system. Int Offshore and Polar Eng Conference, ISOPE, Stavanger, Norway, 2001

[2] Bjornoy $\mathrm{OH}$ and Marley MJ, Assessment of corroded pipelines: Past, Present and Future. Int Offshore and Polar Eng Conference, ISOPE, Stavanger, Norway, 2001

[3] Bjornoy $\mathrm{OH}$, Sigurdsson, G, Marley MJ, Background and development of DNV-RP-F101 "corroded pipelines". Int Offshore and Polar Eng Conference, ISOPE, Stavanger, Norway, 2001

[4] Fu B, Kirkwood MG, Predicting failure pressure of internally corroded linepipe using the finite element method. OMAE, Vol V, 1995, pp 175-184

[5] Batte AD, Fu B, Kirkwood MG and Vu D, New methods for determining the remaining strength of corroded pipelines. OMAE, Vol V, 1997, pp 221-228

[6] Moke DHB, Pick RJ, Glover AG and Hoff R Bursting of line pipe with long external corrosion. Int. J. Pres. Ves. \& Piping, Vol 41, 1991, pp 195-216

[7] Zhu XK and Leis BN, Strength criteria and analytic predictions of failure pressure in corroded line pipes. Int Offshore and Polar Eng Conference, ISOPE, USA, 2003

[8] Zivica V. Utilization of electrical resistance method for the evaluation of the state of steel reinforcement in concrete and the state of its corrosion. Construction and Building Materials, Vol 14, 2000, pp 351-358.

[9] Li Q. Application of probability statistical method to evaluate localized corrosion. Corrosion and Protection, No 1, 1987, pp 32-36.

[10] Xiao Heng, Li Jiuqing, The statistico-analysis for the bit testing data of the Al-Mg alloy. Journal of University of Science and Technology Beijing, Vol 16, No 3, 1994,.pp 97-102 